\title{
Unphosphorylated HSP27 (HSPB1) regulates the translation initiation process via a direct association with eIF4E in osteoblasts
}

\author{
GEN KUROYANAGI $^{1,2}$, HARUHIKO TOKUDA ${ }^{2,3}$, NAOHIRO YAMAMOTO ${ }^{1,2}$, \\ RIE MATSUSHIMA-NISHIWAKI $^{1}$, OSAMU KOZAWA ${ }^{2}$ and TAKANOBU OTSUKA ${ }^{1}$
}

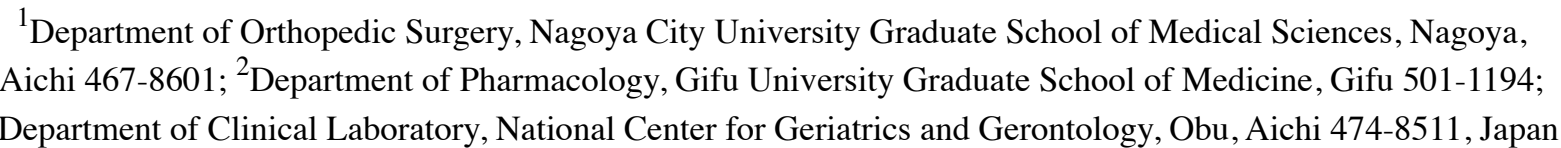

Received January 19, 2015; Accepted June 30, 2015

DOI: $10.3892 /$ ijmm.2015.2274

\begin{abstract}
Heat-shock protein 27 (HSP27/HSPB1) and its phosphorylation are implicated in multiple physiological and pathophysiological cell functions. Our previous study reported that unphosphorylated HSP27 has an inhibitory role in triiodothyronine $\left(\mathrm{T}_{3}\right)$-induced osteocalcin $(\mathrm{OC})$ synthesisin osteoblasts. However, the mechanisms behind the HSP27-mediated effects on osteoblasts remain to be clarified. In the present study, to investigate the exact mechanism of HSP27 and its phosphorylation in osteoblasts, the molecular targets of HSP27 were explored using osteoblast-like MC3T3-E1 cells. The levels of OC mRNA induced by $\mathrm{T}_{3}$ in the HSP27-overexpressing cells did not show any significant differences compared with those in the control empty vector-transfected cells. Therefore, the interactions between HSP27 and translational molecules were focused on, including eukaryotic translation initiation factor 4E (eIF4E), eIF4G and 4E-binding protein 1 (4E-BP1). The HSP27 protein in the unstimulated cells co-immunoprecipitated with eIF4E, but not eIF4G or 4E-BP1. In addition, the association of eIF4E with 4E-BP1 was observed in the HSP27-overexpressing cells, as well as in the control cells. Under $\mathrm{T}_{3}$ stimulation, the binding of eIF4E to eIF4G was markedly attenuated in the HSP27-overexpressing cells compared with the control cells. In addition, the binding of HSP27 to eIF4E in the unstimulated cells was diminished by the phosphorylation of HSP27. In response to $\mathrm{T}_{3}$ stimulation, the association of eIF4E with eIF4G in the unphosphorylatable HSP27-overexpressing cells was markedly reduced compared with the phospho-mimic HSP27-overexpressing cells. Taken together, these findings strongly suggest that unphosphorylated HSP27 associates with eIF4E in osteoblasts and suppresses the translation initiation process.
\end{abstract}

Correspondence to: Dr Osamu Kozawa, Department of Pharmacology, Gifu University Graduate School of Medicine, 1-1 Yanagido, Gifu 501-1194, Japan

E-mail: okkasugai@yahoo.co.jp

Key words: heat-shock protein, eukaryotic translation initiation factor 4E, translation regulation, osteoblast

\section{Introduction}

Heat-shock proteins (HSPs) are induced by a variety of physiological and environmental stresses, such as heat stress (1). As molecular chaperones, HSPs facilitate the refolding of non-native proteins, or assist in their elimination via chaperone-mediated autophagy or via the ubiquitin proteasome system. HSPs have recently been classified into seven families; HSPH (HSP110), HSPC (HSP90), HSPA (HSP70), HSPD/E (HSP60/HSP10), CCT (TRiC), DNAJ (HSP40) and HSPB (small HSP) $(1,2)$. HSP27 (HSPB1) belongs to the HSPB family, with monomeric molecular masses ranging from 15 to $30 \mathrm{kDa}$. The primary structure of HSP27 is highly homologous to other small HSPs, including $\alpha \mathrm{B}$-crystallin and HSP20, which all contain amino acid sequences known as ' $\alpha$-crystallin domains' (3). Although HSP27 is ubiquitously expressed in human cells and tissues, its functions have mainly been studied in skeletal, smooth and cardiac muscles, which have higher expression levels $(1,4)$. The HSP27 expression level is significantly changed under various cellular conditions (1). It is generally recognized that the functions of HSP27 are regulated by post-translational modifications, such as phosphorylation. HSP27, which normally exists as an unphosphorylated oligomer, possesses three phosphorylatable serine residues (Ser-15, Ser-78 and Ser-82) (5,6). Once HSP27 is phosphorylated, a conformational change occurs from the aggregated form to the dimer (5-7). Phosphorylated HSP27 has been previously shown to suppress the growth of hepatocellular carcinoma via inhibition of extracellular signal-regulated kinase (8). However, the exact role of phosphorylated HSP27 has not yet been clarified.

Bone metabolism is strictly regulated through continuous bone remodeling in order to maintain the structural bone integrity and mineral homeostasis (9). Bone remodeling is mainly comprised of two functional events, osteoblastic bone formation and osteoclastic bone resorption (10). It has been reported that the outcome for osteosarcoma patients with overexpression of HSP27 is poorer (11). With regard to the functions of HSP27 in osteoblasts, it is reportedly involved in the balance between differentiation and apoptosis $(12,13)$. The HSP27 protein is barely detectable in osteoblasts under unstimulated conditions (14). In our previous study (15), we 
demonstrated that the level of HSP27 is low in unstimulated osteoblast-like MC3T3-E1 cells. In addition, we reported that various physiological stimuli, including sphingosine-1-phosphate, are able to induce the expression of HSP27 protein in these cells, and that the induced HSP27 is in the unphosphorylated form $(16,17)$. Furthermore, unphosphorylated HSP27 has a suppressive effect on the osteocalcin (OC) synthesis induced by triiodothyronine $\left(\mathrm{T}_{3}\right)$ in MC3T3-E1 cells (17). However, the mechanisms underlying the inhibitory effects of HSP27 on osteoblasts remain to be elucidated.

Evidence is accumulating that HSP27 (HSPB1) interacts with clients regulating gene expression (18). The signal transducer and activator of transcription 2 (STAT2) protein, which is a crucial transcription factor, is reportedly degraded in cells following the downregulation of HSP27, indicating that HSP27 acts as a regulator of transcription (19). In addition, it has been shown that the HSP27-eukaryotic translation initiation factor 4E (eIF4E) interaction decreases eIF4E ubiquitination and proteasomal degradation in advanced prostate cancer cells, and HSP27 has been demonstrated to confer resistance to androgen ablation and chemotherapy through eIF4E (20). It has been firmly established that eIF4E is an mRNA cap-binding protein and has a crucial role in translational control $(21,22)$. eIF4E binds to eIF4G in response to stimulation, and forms the eIF4F complex with eIF4A during the mRNA translation process (23). eIF4E assembles the mRNA into this complex and promotes the ribosome recruitment. In addition, eIF4E is negatively regulated by $4 \mathrm{E}-$ binding protein 1 (4E-BP1), which competes with eIF4G, and under unstimulated conditions, 4E-BP1 binds to eIF4E by occupying the same binding site for eIF4G (24). In the present study, in order to investigate the exact mechanism by which unphosphorylated HSP27 suppresses $\mathrm{T}_{3}$-stimulated $\mathrm{OC}$ synthesis in osteoblasts, the molecular targets of HSP27 were explored using osteoblast-like MC3T3-E1 cells with HSP27 overexpression. Unphosphorylated HSP27 was shown to associate with eIF4E in osteoblasts and suppress the translation initiation process.

\section{Materials and methods}

Materials. $\mathrm{T}_{3}$ and sphingosine-1-phosphate were purchased from Sigma-Aldrich (St. Louis, MO, USA). HSP27, glyceraldehyde-3-phosphate dehydrogenase (GAPDH) and rabbit immunoglobulin $\mathrm{G}(\mathrm{IgG})$ antibodies were purchased from Santa Cruz Biotechnology, Inc. (Dallas, TX, USA). The rabbit monoclonal eIF4E (\#2067), rabbit monoclonal eIF4G (\#5169) and rabbit 4E-BP1 (\#9452) antibodies were purchased from Cell Signaling Technology, Inc. (Danvers, MA, USA). Wild-type (WT) HSP27 and mutant human HSP27s subcloned into the pcDNA3.1(+) mammalian expression vector were kindly provided by Dr C. Schafer (Klinikum Grosshadern, Ludwig-Maximilians University, Munich, Germany). For mutant HSP27 vectors, the HSP27 cDNAs were mutated from serine residues (Ser-15, Ser-78 and Ser-82) to alanine (3A) to prevent the phosphorylation of HSP27, or were mutated to aspartic acid (3D) to imitate the phosphorylated HSP27 form, as described previously (25). The eukaryotic expression vector, pcDNA3.1(+), Dynabeads Protein A and TRIzol reagent were purchased from Life Technologies (Carlsbad, CA, USA). The Omniscript Reverse Transcriptase kit was purchased from Qiagen (Hilden, Germany). FastStart DNA Master SYBR-Green I was obtained from Roche Diagnostics K.K. (Basel, Switzerland). The Bicinchoninic Acid Protein Assay kit was purchased from Thermo Fisher Scientific, Inc. (Waltham, MA, USA). An ECL Western Blotting Detection System was purchased from GE Healthcare UK, Ltd. (Buckinghamshire, UK). Other materials and chemicals were obtained from commercial sources. Sphingosine-1-phosphate was dissolved in dimethyl sulfoxide. The maximum concentration of dimethyl sulfoxide was $0.1 \%$, which did not affect the assay for OC or the detection of protein expression by a western blot analysis.

Cell culture. Cloned osteoblast-like MC3T3-E1 cells derived from newborn mouse calvaria (26) were maintained as described previously (27). Briefly, the cells were cultured in $\alpha$-minimum essential medium ( $\alpha$-MEM) containing $10 \%$ fetal bovine serum (FBS) at $37^{\circ} \mathrm{C}$ in a humidified atmosphere of $5 \% \mathrm{CO}_{2} / 95 \%$ air. The cells were seeded in $35-\mathrm{mm}$ dishes $\left(5 \times 10^{4}\right.$ cells/dish) in $\alpha$-MEM containing $10 \%$ FBS. After 5 days, the medium was exchanged for $\alpha$-MEM containing $0.3 \%$ FBS. The cells were used for experiments after $48 \mathrm{~h}$.

Transient transfections. For transient transfections, the MC3T3-E1 cells were seeded in $35-\mathrm{mm}$ dishes $\left(1.5 \times 10^{4}\right.$ cells/dish) in $\alpha$-MEM containing $10 \%$ FBS. After 4 days, the cultured cells were transfected with $1 \mu \mathrm{g}$ of the WT HSP27 plasmid or control (empty) pcDNA3.1(+) vector using the UniFECTOR transfection reagent (B-Bridge International, Mountain View, CA, USA) in $1 \mathrm{ml}$ of $\alpha$-MEM medium without FBS. Five hours after transfection, $1 \mathrm{ml}$ of medium with $\alpha$-MEM containing $0.6 \%$ FBS was added. At 3 days after transfection, the medium was removed, cells were washed with $2 \mathrm{ml}$ of $\alpha$-MEM medium without FBS, and were incubated in $\alpha$-MEM with $0.3 \%$ FBS for co-immunoprecipitation or reverse transcription-quantitative polymerase chain reaction (RT-qPCR) experiments. The cells were subsequently cultured for another $6 \mathrm{~h}$.

Establishment of stable HSP27-transfected cells. The stable HSP27-transfected cells were established as described previously (17). Briefly, the MC3T3-E1 cells $\left(5 \times 10^{5}\right.$ cells) were transfected with $2 \mu \mathrm{g}$ of the WT, mutant $3 \mathrm{~A}$ or 3D HSP27 plasmids expressing geneticin (G418; EMD Chemicals, Inc., San Diego, CA, USA) resistance using UniFECTOR transfection reagent in $\alpha$-MEM without FBS, and were incubated in the presence of $400 \mu \mathrm{g} / \mathrm{ml}$ of G418. The transfected cells were seeded in $35-\mathrm{mm}$ dishes $\left(5 \times 10^{4}\right.$ cells/dish) in $\alpha$-MEM containing $10 \%$ FBS and $200 \mu \mathrm{g} / \mathrm{ml}$ of G418. After 5 days, the medium was exchanged for $\alpha$-MEM containing $0.3 \%$ FBS and $200 \mu \mathrm{g} / \mathrm{ml}$ of G418. The cells were used for experiments after $48 \mathrm{~h}$.

Protein preparation. For co-immunoprecipitation studies, the cultured MC3T3-E1 cells or transfected cells were lysed in ice-cold TNE lysis buffer $[10 \mathrm{mM}$ Tris/ $\mathrm{HCl}(\mathrm{pH} 7.8), 1 \%$ Nonidet P-40, $150 \mathrm{mM} \mathrm{NaCl}, 1 \mathrm{mM}$ EDTA, $1 \mathrm{mM}$ dithiothreitol, $1 \mathrm{mM}$ sodium fluoride, $1 \mathrm{mM}$ sodium vanadate and protease inhibitor cocktail (Roche Diagnostics K.K.)]. The lysates were subsequently centrifuged at $10,000 \mathrm{xg}$ at $4^{\circ} \mathrm{C}$ 

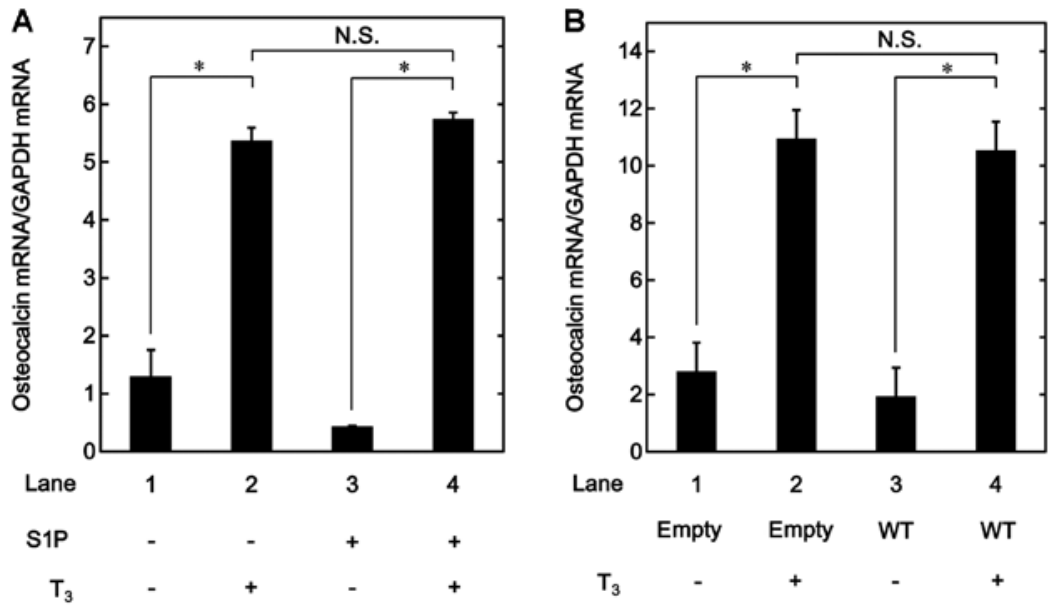

Figure 1. Effects of triiodothyronine $\left(\mathrm{T}_{3}\right)$ on the osteocalcin $(\mathrm{OC})$ mRNA expression in the sphingosine-1-phosphate (S1P)-treated MC3T3-E1 cells and the heat-shock protein 27 (HSP27)-overexpressing cells. (A) The cultured cells were pre-treated with $30 \mu \mathrm{M}$ of S1P or vehicle for $6 \mathrm{~h}$, and subsequently stimulated by $3 \mathrm{nM}$ of $\mathrm{T}_{3}$ or vehicle for $3 \mathrm{~h}$. (B) The cultured cells were transiently transfected with either the control empty vector (empty) or the wild-type (WT) HSP27 vector. The transfected cells were stimulated by $3 \mathrm{nM}$ of $\mathrm{T}_{3}$ or vehicle for $6 \mathrm{~h}$. The respective total RNAs were isolated and transcribed into cDNA. The expression levels of OC mRNA and glyceraldehyde-3-phosphate dehydrogenase (GAPDH) mRNA were quantified by reverse transcription-quantitative polymerase chain reaction. The OC mRNA levels were normalized to those of GAPDH mRNA. Each value represents the mean \pm standard error of the mean of triplicate determinations from three independent cell preparations. ${ }^{*} \mathrm{P}<0.05$ compared to the value of the control. N.S. no significant difference.

for $30 \mathrm{~min}$, and the supernatant was collected as TNE soluble protein. For the western blot analysis of HSP27, eIF4E, eIF4G, 4E-BP1 or IgG, the cultured MC3T3-E1 cells were pre-treated with $30 \mu \mathrm{M}$ of sphingosine-1-phosphate or vehicle for $6 \mathrm{~h}$ to induce HSP27, and were stimulated by $3 \mathrm{nM}$ of $\mathrm{T}_{3}$ or vehicle for $24 \mathrm{~h}$. The HSP27 cDNA-transfected cells were stimulated by $3 \mathrm{nM}$ of $\mathrm{T}_{3}$ or vehicle for $24 \mathrm{~h}$. Following stimulation, the cells were lysed, homogenized and sonicated in lysis buffer containing $62.5 \mathrm{mM}$ Tris/ $\mathrm{HCl}(\mathrm{pH} 6.8), 2 \%$ sodium dodecyl sulfate, $50 \mathrm{mM}$ dithiothreitol and $10 \%$ glycerol.

Co-immunoprecipitation. Co-immunoprecipitation was performed as described previously (28). Briefly, the indicated antibodies were added to the TNE-solubilized proteins, and the mixture was agitated gently overnight at $4^{\circ} \mathrm{C}$, followed by the addition of Dynabeads Protein $\mathrm{A}$ and incubation for a further $1 \mathrm{~h}$ with continuous mixing. Protein immunocomplexes were isolated using a magnetic particle concentrator. The immunoprecipitated proteins and the TNE-soluble proteins (for analysis of the total protein) were analyzed by western blot analysis.

$R T-q P C R$. The cultured MC3T3-E1 cells were pre-treated with $30 \mu \mathrm{M}$ of sphingosine-1-phosphate or vehicle for $6 \mathrm{~h}$ to induce HSP27, and were stimulated by $3 \mathrm{nM}$ of $\mathrm{T}_{3}$ or vehicle in $\alpha$-MEM containing $0.3 \%$ FBS for $3 \mathrm{~h}$. Transiently HSP27-overexpressing cells were stimulated by $3 \mathrm{nM}$ of $\mathrm{T}_{3}$ or vehicle in $\alpha$-MEM containing $0.3 \%$ FBS for $6 \mathrm{~h}$. Total RNA was isolated and transcribed into complementary DNA using the TRIzol reagent and an Omniscript Reverse Transcriptase kit, respectively. RT-qPCR was performed using a LightCycler system in capillaries and the FastStart DNA Master SYBR-Green I provided with the kit. Sense and antisense primers were synthesized based on the report by Zhang et al (29) for mouse OC mRNA and Simpson et al (30) for mouse GAPDH mRNA. The amplified products were determined using a melting curve analysis and agarose electrophoresis. The OC mRNA levels were normalized to those of GAPDH mRNA.
Western blot analysis. A western blot analysis was performed as described previously (17). SDS-PAGE of the prepared cell lysates was performed by the method described by Laemmli (31) in $10 \%$ polyacrylamide gels. The protein was fractionated and transferred onto an Immun-Blot polyvinylidene difluoride (PVDF) membrane (Bio-Rad, Hercules, CA, USA), and a western blot analysis was performed using the indicated primary antibodies with peroxidase-labeled antibodies as secondary antibodies. The primary and secondary antibodies were diluted at 1:1,000 with 5\% skimmed, dried milk in TBST. The peroxidase activity on the PVDF membranes was visualized on X-ray film by means of the ECL Western Blotting Detection System.

Statistical analysis. The data were analyzed by analysis of variance followed by the Bonferroni method for multiple comparisons between pairs, and $\mathrm{P}<0.05$ was considered to indicate a statistically significant difference. All the data are presented as the means \pm standard error of the mean of triplicate determinations from three independent cell preparations.

\section{Results}

Effects of $T_{3}$ on the OC mRNA expression in the sphingosine-1-phosphate-treated MC3T3-E1 cells and the HSP27-overexpressing cells. Our previous study reported that unphosphorylated HSP27 has an inhibitory effect on the OC synthesis induced by $\mathrm{T}_{3}$ in osteoblast-like MC3T3-E1 cells (17). In order to investigate whether the suppressive effect of unphosphorylated HSP27 on $\mathrm{T}_{3}$-stimulated OC release is due to a transcriptional event, the effect of $\mathrm{T}_{3}$ on OC mRNA expression was examined in the MC3T3-E1 cells treated with sphingosine-1-phosphate, which is a physiological inducer of unphosphorylated HSP27 protein expression in these cells, as described in our previous studies $(16,17)$. Sphingosine-1-phosphate, which alone had a limited effect on the levels of OC mRNA, did not affect the increase in the OC mRNA levels stimulated by $\mathrm{T}_{3}$ (Fig. 1A). 
A

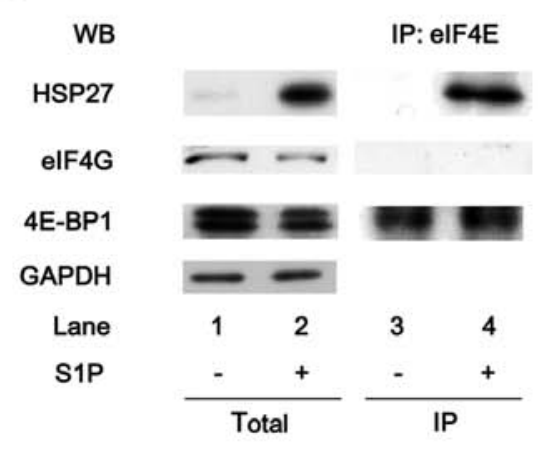

B

WB
HSP27
elF4E
4E-BP1
GAPDH
Lane
S1P

C

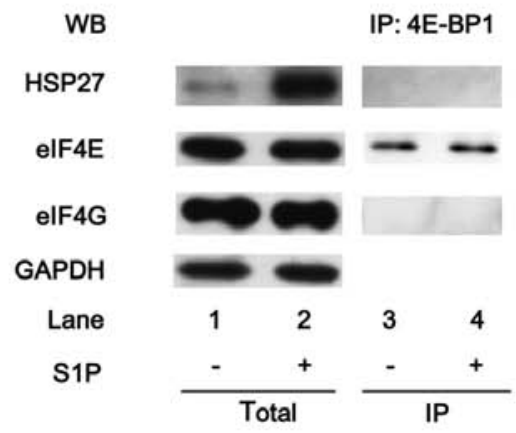

D

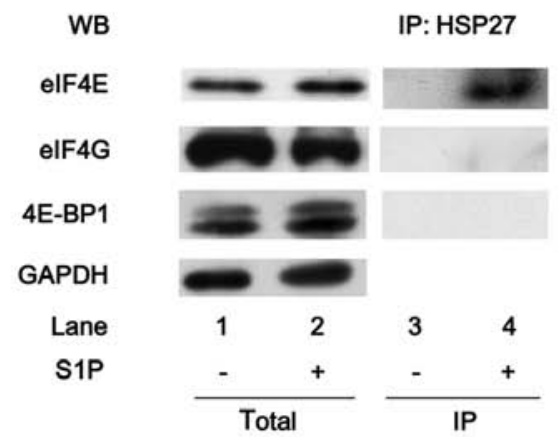

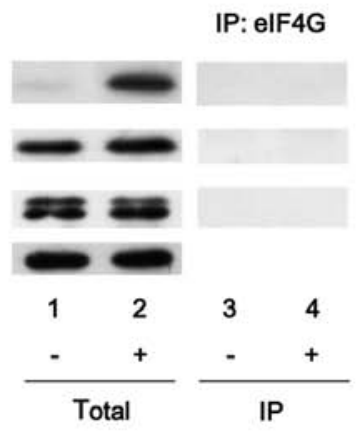

E

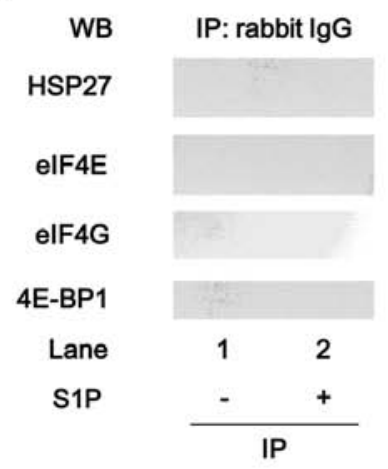

Figure 2. Heat-shock protein 27 (HSP27) interacts with eukaryotic translation initiation factor 4E (eIF4E), but not eIF4G or 4E-binding protein 1 (4E-BP1), in MC3T3-E1 cells with sphingosine-1-phosphate (S1P) treatment. The cultured cells were pre-treated with $30 \mu \mathrm{M}$ of S1P or vehicle for $6 \mathrm{~h}$. (A) The expression levels of HSP27, eIF4G, 4E-BP1 and glyceraldehyde-3-phosphate dehydrogenase (GAPDH) in the pre-immunoprecipitated cell lysates of the cells pre-treated with S1P or vehicle were determined by western blot analysis (total). The cell lysates were immunoprecipitated with eIF4E antibodies, followed by western blot analysis using antibodies for HSP27, eIF4G and 4E-BP1, respectively. (B) The expression levels of HSP27, eIF4E, 4E-BP1 and GAPDH in the pre-immunoprecipitated cell lysates of the cells pre-treated with S1P or vehicle were determined by western blot analysis (total). The cell lysates were immunoprecipitated with eIF4G antibodies, followed by western blot analysis using antibodies for HSP27, eIF4E and 4E-BP1, respectively. (C) The expression levels of HSP27, eIF4E, eIF4G and GAPDH in the pre-immunoprecipitated cell lysates of the cells pre-treated with S1P or vehicle were determined by a western blot analysis (total). The cell lysates were immunoprecipitated with 4E-BP1 antibodies, followed by western blot analysis using antibodies for HSP27, eIF4E and eIF4G, respectively. (D) The expression levels of eIF4E, eIF4G, 4E-BP1 and GAPDH in the pre-immunoprecipitated cell lysates of the cells pre-treated with S1P or vehicle were determined by a western blot analysis (total). The cell lysates were immunoprecipitated with HSP27 antibodies, followed by western blot analysis using antibodies for eIF4E, eIF4G and 4E-BP1, respectively. (E) The cell lysates were immunoprecipitated with normal rabbit IgG, followed by western blot analysis using antibodies for HSP27, eIF4E, eIF4G and 4E-BP1, respectively. IP, immunoprecipitated; WB, western blot analysis.

The effects of $\mathrm{T}_{3}$ on OC mRNA expression were investigated in the HSP27-overexpressing MC3T3-E1 cells, which were transiently transfected with a WT HSP27 plasmid. There were no significant differences between the HSP27-overexpressing cells and the control cells in terms of the mRNA expression levels of $\mathrm{OC}$ stimulated by $\mathrm{T}_{3}$ (Fig. 1B). Our previous study demonstrated that HSP27 in the HSP-overexpressing cells exists in an unphosphorylated form (17).

HSP27 interacts with eIF4E, but not eIF4G or $4 E-B P 1$, in MC3T3-E1 cells with sphingosine-1-phosphate treatment. It is generally established that 4E-BP1 competes with eIF4G and regulates the association of eIF4E and eIF4G to form the active component for the initiation of protein translation (24). HSP27 reportedly interacts with eIF4E, thus resulting in the prevention of eIF4E ubiquitination and proteasomal degradation in advanced prostate cancer cells (20). In addition, it has been shown that HSP27 prevents translation by binding to eIF4G (32). These findings led us to hypothesize that HSP27 regulates the process of translation in osteoblasts. In order to clarify the involvement of HSP27 in the translation initiation process in osteoblast-like MC3T3-E1 cells, whether HSP27 interacts with these molecules, including eIF4E, eIF4G and $4 \mathrm{E}-\mathrm{BP} 1$, was examined. Although the eIF4E protein was co-immunoprecipitated with 4E-BP1 in the unstimulated MC3T3-E1 cells, in which HSP27 was hardly detected, it was not co-immunoprecipitated with HSP27 or eIF4G. By contrast, eIF4E was co-immunoprecipitated with HSP27 in addition to 4E-BP1, but not eIF4G, in the sphingosine-1-phosphate-treated cells (Fig. 2A). eIF4G was not co-immunoprecipitated with HSP27, eIF4E or 4E-BP1 in MC3T3-E1 cells with or without sphingosine-1-phosphate treatment (Fig. 2B). The 4E-BP1 protein was co-immunoprecipitated with eIF4E, but not with HSP27 or eIF4G in these cells treated with or without sphingosine-1-phosphate (Fig. 2C). Additionally, the HSP27 protein was co-immunoprecipitated with eIF4E, but not with eIF4G or 4E-BP1, in MC3T3-E1 cells treated with sphingosine-1-phosphate, whereas it was not co-immunoprecipitated with eIF4E, eIF4G or 4E-BP1 in the unstimulated MC3T3-E1 cells (Fig. 2D). Furthermore, HSP27, eIF4E, eIF4G and 4E-BP1 were not co-immunoprecipitated with normal rabbit IgG (Fig. 2E). 
A

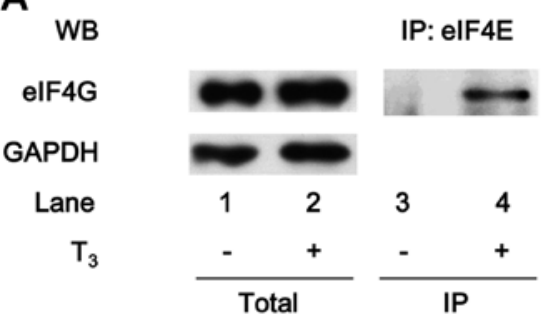

B

WB

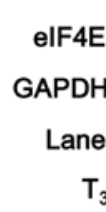

IP: elF4G

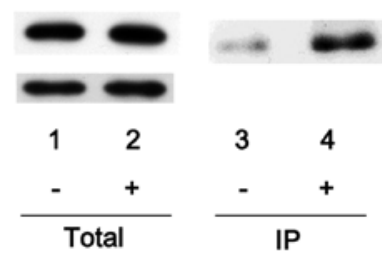

A

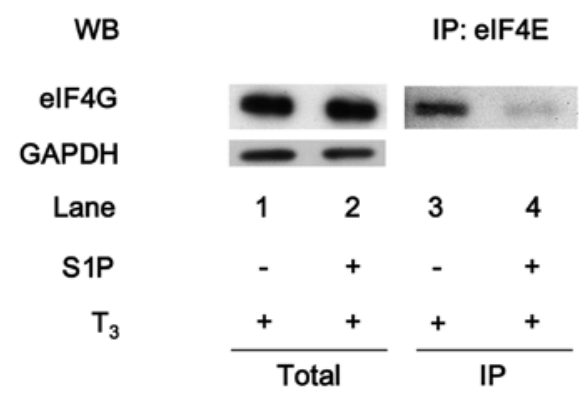

B

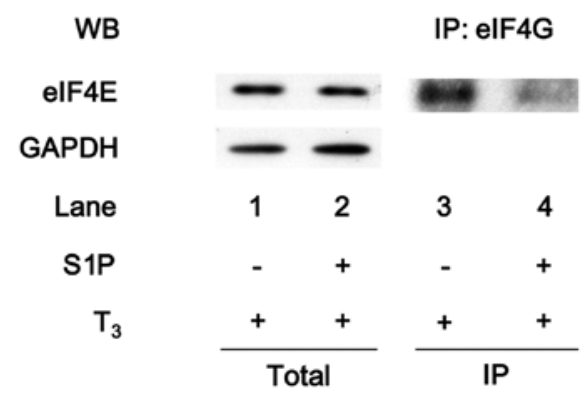

Figure 3. Association of eukaryotic translation initiation factor 4E (eIF4E) with eIF4G under triiodothyronine $\left(\mathrm{T}_{3}\right)$ stimulation in MC3T3-E1 cells. The cultured cells were stimulated by $3 \mathrm{nM}$ of $\mathrm{T}_{3}$ or vehicle for $24 \mathrm{~h}$. (A) The expression levels of eIF4G and glyceraldehyde-3-phosphate dehydrogenase (GAPDH) in the pre-immunoprecipitated cell lysates of the cells stimulated by $\mathrm{T}_{3}$ or vehicle were determined by a western blot analysis (total). The cell lysates were immunoprecipitated with eIF4E antibodies, followed by western blot analysis using eIF4G antibodies. (B) The expression levels of eIF4E and GAPDH in the pre-immunoprecipitated cell lysates of the cells stimulated by $\mathrm{T}_{3}$ or vehicle were determined by a western blot analysis (total). The cell lysates were immunoprecipitated with eIF4G antibodies, followed by western blot analysis using eIF4E antibodies. IP, immunoprecipitated; WB, western blot analysis.

Association of eIF4E with eIF4G under $T_{3}$ stimulation in MC3T3-E1 cells. It is well recognized that eIF4E binds to $4 \mathrm{E}-\mathrm{BP} 1$ under the unstimulated condition, and 4E-BP1 competes with eIF4G for a single binding site on eIF4E (24). In response to stimulation, eIF4E binds to the mRNA $5^{\prime}$ cap structure, mediating the initiation of translation. eIF4E subsequently separates from 4E-BP1 and interacts with eIF4G, which serves as a scaffold protein for the assembly of eIF4E and eIF4A to form the eIF4F complex $(23,24)$. eIF4E was co-immunoprecipitated with eIF4G in the $\mathrm{T}_{3}$-stimulated MC3T3-E1 cells (Fig. 3A). In addition, eIF4G was co-immunoprecipitated with eIF4E in the $\mathrm{T}_{3}$-stimulated cells (Fig. 3B).

Suppression by sphingosine-1-phosphate of the $T_{3}$-induced association of eIF4E with eIF4G in MC3T3-E1 cells. To clarify the effects of HSP27 on translation in osteoblast-like MC3T3-E1 cells, the effect of sphingosine-1-phosphate on the $\mathrm{T}_{3}$-induced binding of eIF4E with eIF4G was examined. As presented in Fig. 3A, the eIF4E protein was co-immunoprecipitated with eIF4G in the $\mathrm{T}_{3}$-stimulated cells. The levels of eIF4E co-immunoprecipitated with eIF4G were markedly reduced in the sphingosine-1-phosphate-treated cells (Fig. 4A). Additionally, the amount of eIF4G protein that co-immunoprecipitated with eIF4E under $\mathrm{T}_{3}$ stimulation was decreased by sphingosine-1-phosphate treatment (Fig. 4B).

HSP27 associates with eIF4E in the HSP27-overexpressing MC3T3-E1 cells. The interaction between HSP27 and translational molecules, such as eIF4E, eIF4G and 4E-BP1, were also examined in the HSP27-overexpressing cells. The expression levels of eIF4E, eIF4G and 4E-BP1 were not different between the HSP27-overexpressing MC3T3-E1 cells and the control cells (Fig. 5A, lanes 1 and 2). In addition, the HSP27 in the cells transiently transfected with WT HSP27 cDNA was an unphosphorylated form (data not shown). The HSP27 protein was co-immunoprecipitated with eIF4E, but not with eIF4G or 4E-BP1, in the HSP27-overexpressing cells (Fig. 5A, lane 4), whereas eIF4E, eIF4G and 4E-BP1 were not co-immunoprecipitated with HSP27 in the control cells (Fig. 5A, lane 3). By contrast, the eIF4E protein in the HSP27-overexpressing cells was co-immunoprecipitated with HSP27 and 4E-BP1 (Fig. 5B, lane 4).

eIF4E does not associate with eIF4G in the HSP27-overexpressing MC3T3-E1 cells with $T_{3}$ stimulation. The association of eIF4E with eIF4G in the HSP27-overexpressing MC3T3-E1 cells under $\mathrm{T}_{3}$ stimulation was further investigated. However, the levels of eIF4E protein co-immunoprecipitated with eIF4G were markedly attenuated in the $\mathrm{T}_{3}$-stimulated WT HSP27-transfected cells compared with the $\mathrm{T}_{3}$-stimulated empty vector-transfected cells (Fig. 6A). In addition, the levels of eIF4G co-immunoprecipitated with eIF4E were decreased 
A

WB

IP: HSP27

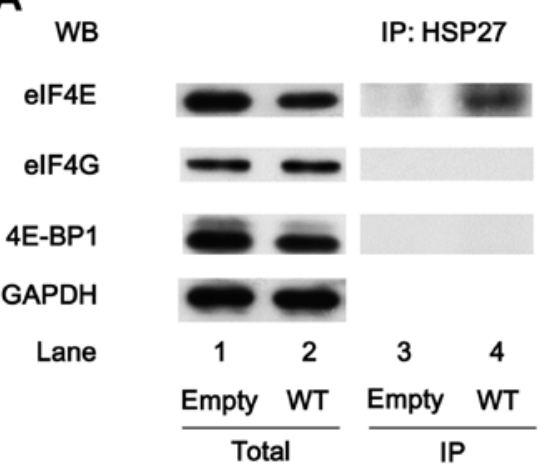

B

WB

HSP27

eIF4G

4E-BP1

GAPDH

Lane

IP: elF4E

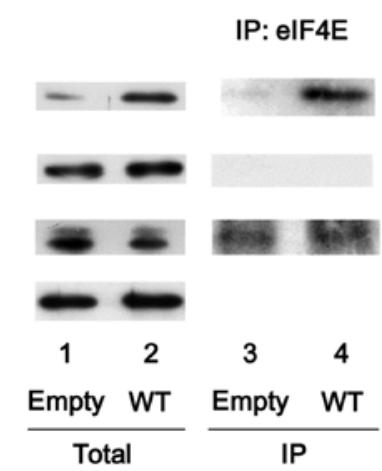

Figure 5. Heat-shock protein 27 (HSP27) associates with eukaryotic translation initiation factor 4E (eIF4E) in the HSP27-overexpressing MC3T3-E1 cells. The cultured cells were transiently transfected with the control empty vector (empty) or the wild-type (WT) HSP27 vector. (A) The expression levels of eIF4E, eIF4G, 4E-binding protein 1 (4E-BP1) and glyceraldehyde-3-phosphate dehydrogenase (GAPDH) in the pre-immunoprecipitated cell lysates of the control cells or WT HSP27-overexpressing cells were determined by western blot analysis (total). The cell lysates were immunoprecipitated with HSP27 antibodies, followed by western blot analysis using antibodies for eIF4E, eIF4G and 4E-BP1, respectively. (B) The expression levels of HSP27, eIF4G, 4E-BP1 and GAPDH in the pre-immunoprecipitated cell lysates of the control cells or the WT HSP27-overexpressing cells were determined by western blot analysis (total). The cell lysates were immunoprecipitated with eIF4E antibodies, followed by western blot analysis using antibodies for HSP27, eIF4G and 4E-BP1, respectively. IP, immunoprecipitated; WB, western blot analysis.

in the WT HSP27-transfected cells under the condition of $\mathrm{T}_{3}$ stimulation (Fig. 6B).

Suppression of the $T_{3}$-induced association of eIF4E with eIF4G in the unphosphorylatable HSP27-overexpressing MC3T3-E1 cells. It is well known that HSP27 undergoes different types of post-translational modifications, such as phosphorylation (1). HSP27 is phosphorylated at three sites (Ser-15, Ser-78 and Ser-82) (5). Previously, we reported that p38 mitogen-activated protein (MAP) kinase is involved in the phosphorylation of HSP27 in the sphingosine-1-phosphate-treated MC3T3-E1 cells (17). Using two stable mutant HSP27-transfected MC3T3-E1 cell lines, the 3A and the 3D HSP27-overexpressing cells, which mimic the unphosphorylated and phosphorylated status of HSP27, respectively, our previous study demonstrated that the $\mathrm{T}_{3}$-stimulated $\mathrm{OC}$ release is significantly decreased in the 3A HSP27-overexpressing cells compared with that in the 3D HSP27-overexpressing cells (17). Therefore, to clarify whether the inhibitory effect of
A

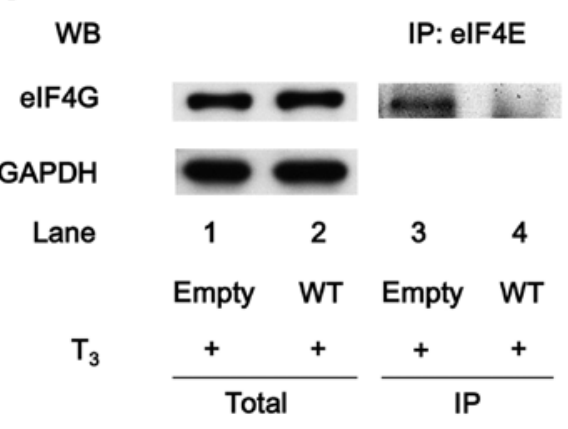

B

WB

IP: eIF4G

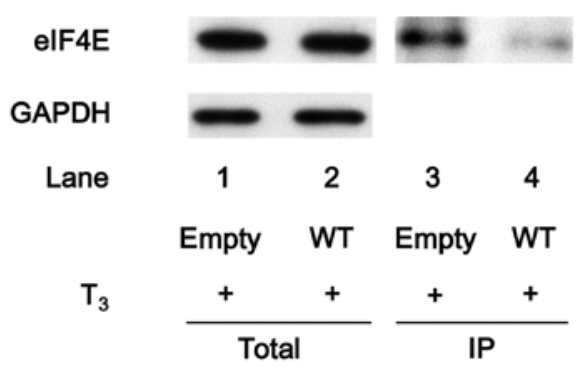

Figure 6. Eukaryotic translation initiation factor 4E (eIF4E) does not associate with eIF4G in the heat-shock protein 27 (HSP27)-overexpressing MC3T3-E1 cells with triiodothyronine $\left(T_{3}\right)$ stimulation. The cultured cells were stably transfected with the control empty vector (empty) or the wild-type (WT) HSP27 vector. Each cell line was stimulated by $3 \mathrm{nM}$ of $\mathrm{T}_{3}$ for $24 \mathrm{~h}$. (A) The expression levels of eIF4G and glyceraldehyde-3-phosphate dehydrogenase (GAPDH) in the pre-immunoprecipitated cell lysates of the control cells or the HSP27-overexpressing cells were determined by a western blot analysis (total). The cell lysates were immunoprecipitated with eIF4E antibodies, followed by western blot analysis using eIF4G antibodies. (B) The expression levels of eIF4E and GAPDH in the pre-immunoprecipitated cell lysates of the control cells or the HSP27-overexpressing cells were determined by a western blot analysis (total). The cell lysates were immunoprecipitated with eIF4G antibodies, followed by western blot analysis using eIF4E antibodies. IP, immunoprecipitated; WB, western blot analysis.

unphosphorylated HSP 27 on the $\mathrm{T}_{3}$-stimulated OC synthesis is due to the suppression of the association of eIF4E with eIF4G, the binding of eIF4E and eIF4G under stimulation by $\mathrm{T}_{3}$ in these two types of mutant HSP27-transfected MC3T3-E1 cells was examined. The eIF4E protein was co-immunoprecipitated with eIF4G in the 3D HSP27-overexpressing cells stimulated by $\mathrm{T}_{3}$ (Fig. 7A, lane 3 ). However, under $\mathrm{T}_{3}$ stimulation, the levels of eIF4E co-immunoprecipitated with eIF4G were markedly lower in the 3A HSP27-overexpressing cells than those in the 3D HSP27-overexpressing cells (Fig. 7A, lane 4). Furthermore, the levels of eIF4G co-immunoprecipitated with eIF4E were decreased in the $\mathrm{T}_{3}$-stimulated 3A HSP27-overexpressing cells in comparison with those in the $\mathrm{T}_{3}$-stimulated $3 \mathrm{D}$ HSP27-overexpressing cells (Fig. 7B).

\section{Discussion}

In the present study, the molecular targets of HSP27 were investigated using osteoblast-like MC3T3-E1 cells, on the basis of our previous study, which showed that unphosphorylated, but not phosphorylated, HSP27 acts as a negative regulator in $\mathrm{T}_{3}$-induced OC synthesis in these cells (17). Therefore, whether 
A

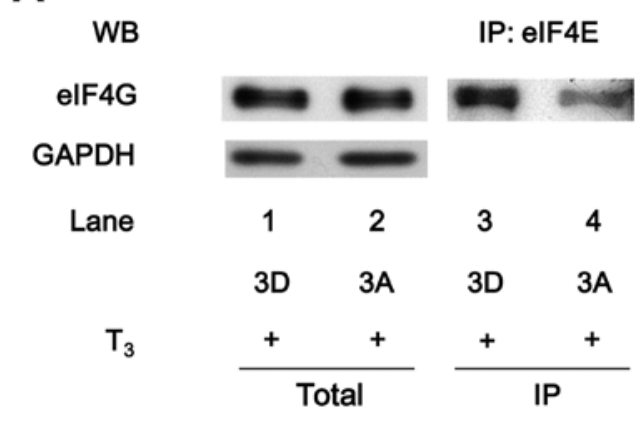

B

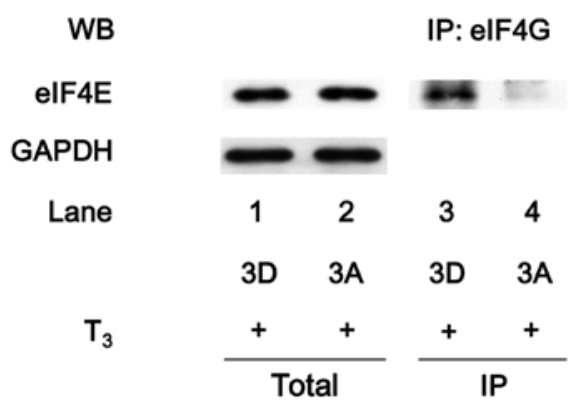

Figure 7. Suppression of the triiodothyronine $\left(\mathrm{T}_{3}\right)$-induced association of eukaryotic translation initiation factor 4E (eIF4E) with eIF4G in the unphosphorylatable heat-shock protein 27 (HSP27)-overexpressing MC3T3-E1 cells The cultured cells were stably transfected with either the phospho-mimic (3D) or the unphosphorylatable (3A) HSP27 vectors. The cells were stimulated by $3 \mathrm{nM}$ of $\mathrm{T}_{3}$ for $24 \mathrm{~h}$. (A) The expression levels of eIF4G and glyceraldehyde-3-phosphate dehydrogenase (GAPDH) in the pre-immunoprecipitated cell lysates of the phospho-mimic HSP27-overexpressing cells or the unphosphorylatable HSP27-overexpressing cells were determined by western blot analysis (total). The cell lysates were immunoprecipitated with eIF4E antibodies, followed by western blot analysis using eIF4G antibodies (B) The expression levels of eIF4E and GAPDH in the pre-immunoprecipitated cell lysates of the phospho-mimic HSP27-overexpressing cells or the unphosphorylatable HSP27-overexpressing cells were determined by western blot analysis (total). The cell lysates were immunoprecipitated with eIF4G antibodies, followed by western blot analysis using eIF4E antibodies. IP, immunoprecipitated; WB, western blot analysis.

the OC mRNA expression levels stimulated by $\mathrm{T}_{3}$ are affected in MC3T3-E1 cells in which HSP27 expression is induced was examined. The $\mathrm{T}_{3}$-stimulated $\mathrm{OC}$ mRNA expression levels were hardly affected in the MC3T3-E1 cells pre-treated with sphingosine-1-phosphate, a physiological sphingomyelin metabolite, which is capable of inducing the unphosphorylated form of HSP27 in these cells, compared with the cells without sphingosine-1-phosphate pre-treatment. In addition, the expression levels of OC mRNA induced by $\mathrm{T}_{3}$ did not show any significant differences between the WT HSP27-transfected cells and the empty vector-transfected cells. The HSP27 in the HSP27-overexpressing MC3T3-E1 cells exists in an unphosphorylated form. Based on these findings, it appears unlikely that the suppressive effects of unphosphorylated HSP27 on the $\mathrm{T}_{3}$-induced $\mathrm{OC}$ synthesis are exerted at a point upstream of transcription in osteoblast-like MC3T3-E1 cells.

The association between HSP27 and molecules involved in translation, including eIF4E, eIF4G and 4E-BP1, was investigated. During the mRNA translation process, eIF4E has a crucial role as the mRNA cap-binding protein (21). In response to stimulation, eIF4E binds to the mRNA 5' cap structure and forms the eIF4F complex with its cofactors, eIF4G and eIF4A. The eIF4F complex contributes to the ribosomal recruitment of mRNA, the rate-limiting step in translation initiation (21-23). The eIF4E protein was co-immunoprecipitated with 4E-BP1, but not with eIF4G, in the unstimulated MC3T3-E1 cells without HSP27 induction by sphingosine-1-phosphate. In the unstimulated cells with sphingosine-1-phosphate pre-treatment, eIF4E was co-immunoprecipitated with not only 4E-BP1, but also HSP27. Additionally, the HSP27 protein was markedly co-immunoprecipitated with eIF4E, but not with eIF4G or 4E-BP1, and 4E-BP1 was co-immunoprecipitated with eIF4E, but not HSP27. Furthermore, a similar phenomenon was observed regarding the interaction of HSP27 with eIF4E, eIF4G and 4E-BP1 in the MC3T3-E1 cells that had been transfected with the WT HSP27 vector. Thus, it is possible that HSP27 may bind to eIF4E instead of 4E-BP1. Therefore, these findings suggest that eIF4E exists as two independent forms, a 4E-BP1- and a HSP27-associated form, in unstimulated MC3T3-E1 cells with HSP27 induction, whereas eIF4E exists as one form, a 4E-BP1-associated form, in the cells without HSP27 induction.

It is firmly established that 4E-BP1 separates from eIF4E, and subsequently eIF4G binds to eIF4E at the same binding site as 4E-BP1 under the stimulated condition (24). The association of eIF4E with eIF4G in osteoblast-like MC3T3-E1 cells was found to increase in response to $\mathrm{T}_{3}$ stimulation. In order to clarify the role of HSP27 in the initiation of translation in osteoblast-like MC3T3-E1 cells, the binding of eIF4E and eIF4G in the $\mathrm{T}_{3}$-stimulated cells with sphingosine-1-phosphate pre-treatment was examined. The levels of eIF4E co-immunoprecipitated with eIF4G were markedly attenuated in the MC3T3-E1 cells pre-treated with sphingosine-1-phosphate. The association of eIF4E with eIF4G in the $\mathrm{T}_{3}$-stimulated MC3T3-E1 cells transfected with the WT HSP27 vector was further investigated, and the levels of eIF4E co-immunoprecipitated with eIF4G in these cells were much weakened compared with those in the empty vector-transfected cells. Our previous study showed that the HSP27 in the MC3T3-E1 cells transfected with WT HSP27 vector is an unphosphorylated form (17). Taking all these findings into account, it is most likely that unphosphorylated HSP27 attenuates eIF4E-eIF4G binding under $\mathrm{T}_{3}$ stimulation, resulting in the downregulation of the translation initiation process in osteoblasts.

HSP27 (HSPB1) is currently known to undergo several types of post-translational modifications, including phosphorylation, suggesting that the modifications alter the HSP27 functions (5). Therefore, whether the phosphorylation of HSP27 affects its binding to eIF4E in osteoblast-like MC3T3-E1 cells was investigated. Our previous study reported that HSP27 is phosphorylated via p38 MAP kinase activation in sphingosine-1-phosphate-induced MC3T3-E1 cells (17). Using two mutant HSP27-transfected MC3T3-E1 cell lines, unphosphorylatable HSP27-overexpressing cells (3A) and phospho-mimic HSP27-overexpressing cells (3D), eIF4E was markedly co-immunoprecipitated with eIF4G in 3D cells stimulated by $\mathrm{T}_{3}$, whereas the levels of eIF4E co-immunoprecipitated with eIF4G were reduced in the $\mathrm{T}_{3}$-stimulated $3 \mathrm{~A}$ cells. Our previous study reported that the OC release induced by $\mathrm{T}_{3}$ in $3 \mathrm{~A}$ cells is suppressed in comparison with that 


\section{Without HSP27 induction}

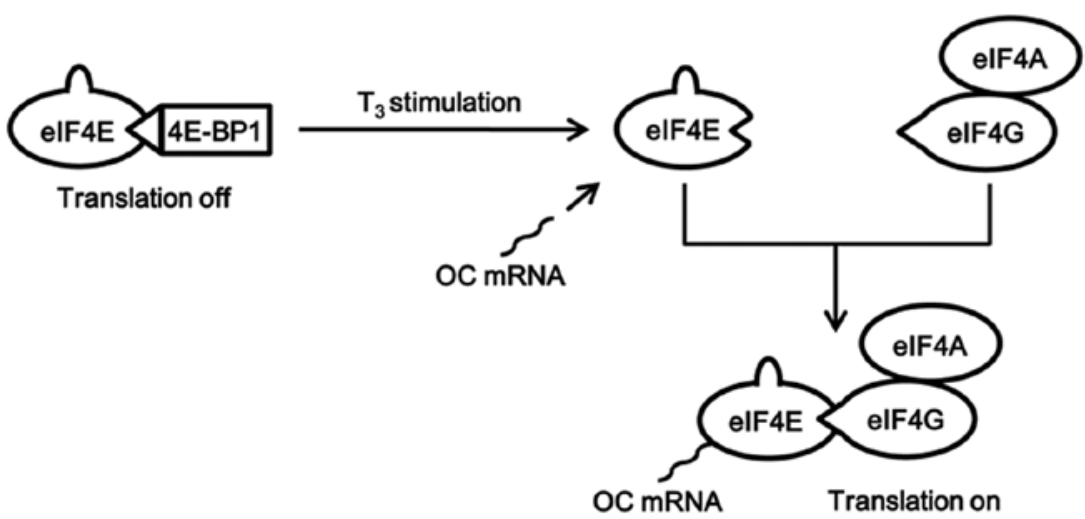

II. With HSP27 induction

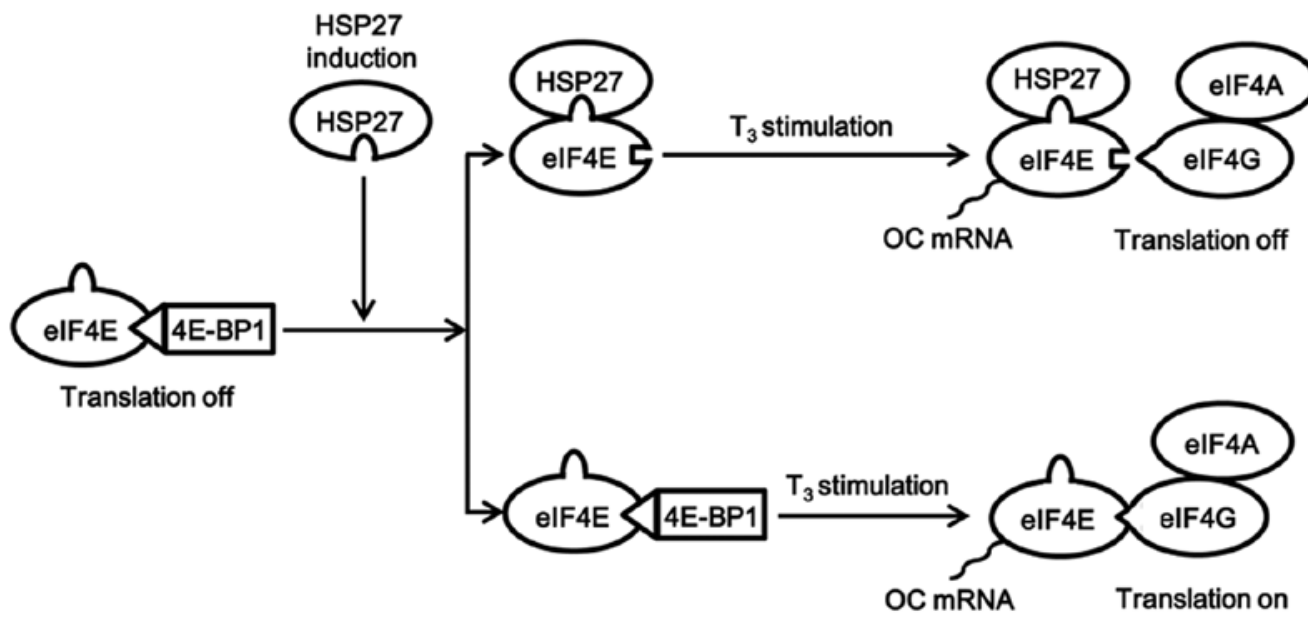

Figure 8. Potential mechanism underlying the triiodothyronine $\left(\mathrm{T}_{3}\right)$-induced osteocalcin $(\mathrm{OC})$ synthesis with or without heat-shock protein 27 (HSP27) induction in osteoblast-like MC3T3-E1 cells. eIF, eukaryotic translation initiation factor; 4E-BP1, 4E-binding protein 1.

in the 3D cells (17). Taking these results into account, it is most likely that unphosphorylated, but not phosphorylated, HSP27 associates with eIF4E under the unstimulated condition in osteoblast-like MC3T3-E1 cells, and that the formation of the HSP27-eIF4E complex downregulates the translation initiation process under stimulation via suppression of the eIF4E-eIF4G interaction. It is possible that the induction of HSP27 and its phosphorylation have a critical role in the translation process of $\mathrm{T}_{3}$-stimulated OC synthesis in osteoblast-like MC3T3-E1 cells. A schematic illustration of the potential mechanism underlying these effects of HSP27 is presented in Fig. 8.

It is well known that HSP27 normally exists in an aggregated form, but when it is phosphorylated, a conformational change occurs that leads to the formation of dimers (5-7). Our previous study demonstrated that HSP27 reduces the release of vascular endothelial growth factor (VEGF) induced by TGF- $\beta$ via a post-transcriptional mechanism in osteoblast-like MC3T3-E1 cells in which HSP27 expression is induced (33). In addition, phosphorylated HSP27 changes its localization from the cytosol to the perinuclear region in these cells, and acts as a functional regulator of the endoplasmic reticulum, contributing to the modulation of $\mathrm{T}_{3}$-induced $\mathrm{OC}$ synthesis (17). Taking all of these findings into account, it is possible that the change in the conformation or localization of HSP27 by its phosphorylation contributes to the regulation of the translation initiation process, thereby affecting the synthesis of proteins, such as OC and VEGF in osteoblasts. Phosphorylated HSP27 is reportedly involved in the pathogenesis of conditions, such as diabetic kidney disease and viral infections $(34,35)$. Further investigation is necessary to clarify the precise role of HSP27 and its phosphorylation status in osteoblasts.

In conclusion, the present results strongly suggest that the phosphorylation status of HSP27 has a role in switching its binding to eIF4E, resulting in regulation of the translation initiation process in osteoblasts.

\section{Acknowledgements}

The authors are grateful to Dr Yumiko Kurokawa for her skillful technical assistance. The present study was supported in part by a Grant-in-Aid for Scientific Research (no. 19591042) from the Ministry of Education, Culture, Sports, Science and 
Technology of Japan and the Research Funding for Longevity Sciences (nos. 23-9 and 25-4) from the National Center for Geriatrics and Gerontology (NCGG) of Japan.

\section{References}

1. Mymrikov EV, Seit-Nebi AS and Gusev NB: Large potentials of small heat shock proteins. Physiol Rev 91: 1123-1159, 2011.

2. Kampinga HH, Hageman J, Vos MJ, Kubota H, Tanguay RM, Bruford EA, Cheetham ME, Chen B and Hightower LE: Guidelines for the nomenclature of the human heat shock proteins. Cell Stress Chaperones 14: 105-111, 2009.

3. Kriehuber T, Rattei T, Weinmaier T, Bepperling A, Haslbeck M and Buchner J: Independent evolution of the core domain and its flanking sequences in small heat shock proteins. FASEB J 24: 3633-3642, 2010.

4. Dubińska-Magiera M, Jabłońska J, Saczko J, Kulbacka J, Jagla T and Daczewska M: Contribution of small heat shock proteins to muscle development and function. FEBS Lett 588: 517-530, 2014

5. Kostenko S and Moens U: Heat shock protein 27 phosphorylation: Kinases, phosphatases, functions and pathology. Cell Mol Life Sci 66: 3289-3307, 2009.

6. Landry J, Lambert H, Zhou M, Lavoie JN, Hickey E, Weber LA and Anderson CW: Human HSP27 is phosphorylated at serines 78 and 82 by heat shock and mitogen-activated kinases that recognize the same amino acid motif as S6 kinase II. J Biol Chem 267: 794-803, 1992.

7. Hayes D, Napoli V, Mazurkie A, Stafford WF and Graceffa P: Phosphorylation dependence of hsp27 multimeric size and molecular chaperone function. J Biol Chem 284: 18801-18807, 2009.

8. Matsushima-Nishiwaki R, Takai S, Adachi S, Minamitani C, Yasuda E, Noda T, Kato K, Toyoda H, Kaneoka Y Yamaguchi A, et al: Phosphorylated heat shock protein 27 represses growth of hepatocellular carcinoma via inhibition of extracellular signal-regulated kinase. J Biol Chem 283: 18852-18860, 2008

9. Kular J, Tickner J, Chim SM and Xu J: An overview of the regulation of bone remodelling at the cellular level. Clin Biochem 45: $863-873,2012$

10. Chim SM, Tickner J, Chow ST, Kuek V, Guo B, Zhang G, Rosen V, Erber W and Xu J: Angiogenic factors in bone local environment. Cytokine Growth Factor Rev 24: 297-310, 2013.

11. Uozaki $H$, Horiuchi $H$, Ishida $T$, Iijima $T$, Imamura $T$ and Machinami R: Overexpression of resistance-related proteins (metallothioneins, glutathione-S-transferase pi, heat shock protein 27, and lung resistance-related protein) in osteosarcoma Relationship with poor prognosis. Cancer 79: 2336-2344, 1997.

12. Tiffee JC, Griffin JP and Cooper LF: Immunolocalization of stress proteins and extracellular matrix proteins in the rat tibia. Tissue Cell 32: 141-147, 2000.

13. Leonardi R, Barbato E, Paganelli C and Lo Muzio L: Immunolocalization of heat shock protein 27 in developing jaw bones and tooth germs of human fetuses. Calcif Tissue Int 75: 509-516, 2004.

14. Shakoori AR, Oberdorf AM, Owen TA, Weber LA, Hickey E, Stein JL, Lian JB and Stein GS: Expression of heat shock genes during differentiation of mammalian osteoblasts and promyelocytic leukemia cells. J Cell Biochem 48: 277-287, 1992.

15. Kozawa O, Niwa M, Matsuno H, Ishisaki A, Kato $\mathrm{K}$ and Uematsu T: Stimulatory effect of basic fibroblast growth factor on induction of heat shock protein 27 in osteoblasts: Role of protein kinase C. Arch Biochem Biophys 388: 237-242, 2001.

16. Kozawa O, Niwa M, Matsuno $H$, Tokuda $H$, Miwa M, Ito $H$, Kato $\mathrm{K}$ and Uematsu T: Sphingosine 1-phosphate induces heat shock protein 27 via p38 mitogen-activated protein kinase activation in osteoblasts. J Bone Miner Res 14: 1761-1767, 1999.

17. Kato K, Adachi S, Matsushima-Nishiwaki R, Minamitani C, Natsume H, Katagiri Y, Hirose Y, Mizutani J, Tokuda H, Kozawa O, et al: Regulation by heat shock protein 27 of osteocalcin synthesis in osteoblasts. Endocrinology 152: 1872-1882, 2011.
18. Arrigo AP and Gibert B: HspB1, HspB5 and HspB4 in human cancers: Potent oncogenic role of some of their client proteins. Cancers (Basel) 6: 333-365, 2014.

19. Gibert B, Eckel B, Fasquelle L, Moulin M, Bouhallier F, Gonin V, Mellier G, Simon S, Kretz-Remy C, Arrigo AP, et al: Knock down of heat shock protein 27 (HspB1) induces degradation of several putative client proteins. PLoS One 7: e29719, 2012.

20. Andrieu C, Taieb D, Baylot V, Ettinger S, Soubeyran P, De-Thonel A, Nelson C, Garrido C, So A, Fazli L, et al: Heat shock protein 27 confers resistance to androgen ablation and chemotherapy in prostate cancer cells through eIF4E. Oncogene 29: 1883-1896, 2010.

21. Kong $\mathbf{J}$ and Lasko P: Translational control in cellular and developmental processes. Nat Rev Genet 13: 383-394, 2012.

22. Gilbert RJ, Gordiyenko Y, von der Haar T, Sonnen AF, Hofmann G, Nardelli M, Stuart DI and McCarthy JE: Reconfiguration of yeast $40 \mathrm{~S}$ ribosomal subunit domains by the translation initiation multifactor complex. Proc Natl Acad Sci USA 104: 5788-5793, 2007.

23. Sonenberg $\mathrm{N}$ and Gingras AC: The mRNA 5' cap-binding protein IF4E and control of cell growth. Curr Opin Cell Biol 10: 268-275, 1998 .

24. Jia Y, Polunovsky V, Bitterman PB and Wagner CR: Cap-dependent translation initiation factor eIF4E: An emerging anticancer drug target. Med Res Rev 32: 786-814, 2012.

25. Kubisch C, Dimagno MJ, Tietz AB, Welsh MJ, Ernst SA, Brandt-Nedelev B, Diebold J, Wagner AC, Göke B, Williams JA, et al: Overexpression of heat shock protein Hsp27 protects against cerulein-induced pancreatitis. Gastroenterology 127: 275-286, 2004.

26. Sudo H, Kodama HA, Amagai Y, Yamamoto S and Kasai S: In vitro differentiation and calcification in a new clonal osteogenic cell line derived from newborn mouse calvaria. J Cell Biol 96: 191-198, 1983.

27. Kozawa O, Tokuda H, Miwa M, Kotoyori J and Oiso Y: Cross-talk regulation between cyclic AMP production and phosphoinositide hydrolysis induced by prostaglandin $\mathrm{E}_{2}$ in osteoblast-like cells. Exp Cell Res 198: 130-134, 1992.

28. Matsushima-Nishiwaki R, Kumada T, Nagasawa T, Suzuki M, Yasuda E, Okuda S, Maeda A, Kaneoka Y, Toyoda $\mathrm{H}$ and Kozawa O: Direct association of heat shock protein 20 (HSPB6) with phosphoinositide 3-kinase (PI3K) in human hepatocellular carcinoma: Regulation of the PI3K activity. PLoS One 8: e78440, 2013.

29. Zhang W, Yang N and Shi XM: Regulation of mesenchymal stem cell osteogenic differentiation by glucocorticoid-induced leucine zipper (GILZ). J Biol Chem 283: 4723-4729, 2008.

30. Simpson DA, Feeney S, Boyle C and Stitt AW: Retinal VEGF mRNA measured by SYBR green I fluorescence: A versatile approach to quantitative PCR. Mol Vis 6: 178-183, 2000.

31. Laemmli UK: Cleavage of structural proteins during the assembly of the head of bacteriophage T4. Nature 227: 680-685, 1970.

32. Cuesta R, Laroia G and Schneider RJ: Chaperone hsp27 inhibits translation during heat shock by binding eIF4G and facilitating dissociation of cap-initiation complexes. Genes Dev 14: 1460-1470, 2000.

33. Kato K, Tokuda H, Adachi S, Matsushima-Nishiwaki R, Yamauchi J, Natsume H, Minamitani C, Mizutani J, Otsuka T and Kozawa O: Role of heat shock protein 27 in transforming growth factor- $\beta$-stimulated vascular endothelial growth factor release in osteoblasts. Int J Mol Med 27: 423-428, 2011.

34. Barutta F, Pinach S, Giunti S, Vittone F, Forbes JM, Chiarle R, Arnstein M, Perin PC, Camussi G, Cooper ME, et al: Heat shock protein expression in diabetic nephropathy. Am J Physiol Renal Physiol 295: F1817-F1824, 2008.

35. Singh D, McCann KL and Imani F: MAPK and heat shock protein 27 activation are associated with respiratory syncytial virus induction of human bronchial epithelial monolayer disruption. Am J Physiol Lung Cell Mol Physiol 293: L436-L445, 2007. 